\title{
RESEARCH REVIEW FOR DIGITAL IMAGE SEGMENTATION TECHNIQUES
}

\author{
Ashraf A. Aly ${ }^{1}$, Safaai Bin Deris ${ }^{2}$, Nazar Zaki $^{3}$ \\ ${ }^{1,2}$ Faculty of Computer Science, Universiti Teknologi Malaysia \\ Ashraf.ahmed@uaeu.ac.ae, safaai@utm.my \\ ${ }^{3}$ College of Information Technology, UAE University, UAE \\ nzaki@uaeu.ac.ae
}

\begin{abstract}
Evaluating the previous work is an important part of developing segmentation methods for the image analysis techniques. The aim of this paper is to give a review of digital image segmentation techniques. The problems of digital image segmentation represent great challenges for computer vision. The wide range of the problems of computer vision may make good use of image segmentation. This paper study and evaluate the different methods for segmentation techniques. We discuss the main tendency of each algorithm with their applications, advantages and disadvantages. This study is useful for determining the appropriate use of the image segmentation methods and for improving their accuracy and performance and also for the main objective, which designing new algorithms.
\end{abstract}

\section{KEYWORDS}

Active Contour, Segmentation Enhancement, Topological Alignments, Boundary Detection, image Segmentation, Inversion Technique.

\section{INTRODUCTION}

Digital image processing is important domain for many reasons. Actually Digital image processing is a recent subject in computer history. In 1960s; Bell Labs and University of Maryland, and a few other places started to develop several techniques for digital image processing. With application to satellite imagery, wire photo standards conversion, medical imaging, videophone, character recognition, and photo enhancement. But the cost of processing was fairly high with the computing equipment of that era. In the 1970s, image processing proliferated, when cheaper computers and dedicated hardware became available. Images could then be processed in real time, for some dedicated problems such as television standards conversion. As general-purpose computers became faster, they started to take over the role of dedicated hardware for all but the most specialized and compute-intensive operations.

In digital image processing, we use computer algorithms to perform image processing. Actually digital image processing has several advantages over the analog image processing; first it gives a high number of algorithms to be used with the input data, second we can avoid some processing problems such as creating noise and signal distortion during signal processing. In 2000s, fast computers became available for signal processing and digital image processing has become the popular form of image processing. Because of that, signal image processing became versatile method, and also cheapest.

Image segmentation is important part in many signal processing technique and its applications. The segmentation procedure is to find the better positions of the shape points according to the 
appearance information. Algorithms based on classifiers have been widely applied to segment organs in medical images like cardiac and brain images. The goal of image segmentation process is partitioning the image into regions. Image segmentation applications identifying objects in a scene for object-based measurements such as size and shape, identifying objects in a moving scene for object-based video compression, identifying objects which are at different distances from a sensor using depth measurements from a laser range finder enabling path planning for mobile robots. The purpose of image segmentation is to cluster pixels of an image into image regions. We can use segmentation for image compression, object recognition, and image editing processing.

\section{RELATED WORK}

The problems of digital image segmentation represent great challenges for computer vision. The wide range of the problems of computer vision may make good use of image segmentation. Many researchers had created several methods to deal with the problem of image segmentation. Zimmer et al. [10] created a method to detect the mobility of live cells using the active contour (snakes) method. Mukherjee et al. [30] modified a method to handle the tracking problem using threshold method. Coskun et al. [4] used the inverse modelling to detect the mobility of living cells. Recently there have been a number of researchers has tried to create several image segmentation algorithms as in (Krinidis et al., Mélange, et al., Mignotte et al.).

In this paper we managed to review and summarized the major techniques for digital image segmentation. We categorized these techniques based on the segmentation method which the technique is using. In Table 2.1 a comparison of the image segmentation methods.

\section{DIGITAL IMAGE SEGMENTATION METHODS}

In the following subsection, we will review the methods applied to segment images.

The segmentation of an image $I$, which represent a set of pixels is partitioning into $n$ disjoint sets $R 1, R 2, \ldots, R n$, called segments or regions such that their union of all regions equals $I$, $\mathrm{I}=\mathrm{R}_{1} \mathrm{U} \mathrm{R}_{2} \mathrm{U} \ldots \ldots \mathrm{U} \mathrm{R}_{\mathrm{n}}$.

\subsection{Inversion technique}

The principle of the inversion is to continuously update the muscle activity to produce a face movement following a given face trajectory (Smith et al., 1986; Terzopoulos et al., 1991; Press et al., 1992; Waters et al., 1996). When the inversion had been carried out for all frames, the inverted activity was used to generate an animation. A conventional nonlinear optimizer minimizing a cost function was selected to implement the inversion. The cost function $E$ was the sum of the squares of the Euclidean distances between the markers and the corresponding face model nodes:

$E=\sum_{i=1}^{N}\left|m_{i}-n_{i}\right|^{2}$

where $m i$ and $n i$ are the 3-D positions of the $i$ th marker and face model node, respectively, $N$ is the number of nodes used in the inversion, and ||$^{2}$ is the vectorial magnitude square operator, i.e., the sum of the squares of each coordinate of the vector.

The inversion could produce different activity patterns, depending on the initial conditions. Constraints may be added to the inversion to limit the number of solutions. In all analyses, the 
inversion was carried out without constraints; then with the constraint that the inverted filtered EMG values had to be positive. The new positive constraint cost function $E^{\prime}$ was redefined in the second case by:

$$
\begin{aligned}
& E^{\prime}=\sum_{i=1}^{N}\left|m_{i}-n_{i}\right|^{2}, \text { if all filtered EMG } \geq 0 \\
& E^{\prime}=10^{6}\left(1+\left|\sum E M G 0\right|\right), \text { if at least one filtered EMG } \geq 0
\end{aligned}
$$

Where $m_{i}$ and $n_{i}$ are the 3-D positions of the $i^{\text {th }}$ marker and face model node, respectively, $N$ is the number of nodes used in the inversion, and EMG0 is the set of negative muscle activity levels. The constraint that all filtered EMG had to be greater than zero will be called the positive constraint. The advantage of the method is the high quality of the animation and also the data is very good, but the different EMG patterns can produce the same kinematic output which affects the accuracy.

\subsection{Pattern Recognition Techniques}

Pattern Recognition Techniques is a non-linear modelling tools and we can be used to model the inputs and outputs relationships (fukunaga et al., 1990; Awwal et al., 1992; Portegys et al., 1995; Coskun et al., 2007; Baum et al., 1998). Weights in the classifier are selected through optimizing energy functional defined by the features of structures and are updated through processing each sample in the training set.

The extracted information from the training set provides important cues of the structures such as intensity, position and shape, which can be valuable complementary information for the segmentation of test images. Active appearance models (AAM) are statistical models of the shape of structures. Training samples are used to extract the mean shape, mean appearance and define ranges of shape parameters. Restrictions on shape parameters guarantee the similarity between the segmentation result and the training samples. The segmentation procedure is to find the better positions of the shape points according to the appearance information. Algorithms based on classifiers have been widely applied to segment organs in medical images like cardiac and brain images. If properly modelled, supervised classification algorithms can greatly enhance the segmentation accuracy. However, supervised classification algorithms are sensitive to the initial conditions. To guarantee the correctness of the results, the training set must contain enough samples and the samples should be representative and segmented accurately.

\subsection{Active contour models}

Active contour models (snakes) goal is to apply segmentation process to an image by doing deformation to the initial contour towards the boundary of the object of interest. We do that by deforming an initial contour to minimizing the energy function which defined on contours (Kass et al. 1987; Ray et al., 2002; Zimmer et al., 2002; Kruse et al., 1996; Sacan et al., 2008). There are two components in this energy: the potential energy, which is small when the contour is aligned to edges of the image, and the internal deformation energy, which is small when the contour is smooth. Both components are contour integrals with respect to a parameter of the contour.

An Active contour can be parametrically represented by $\mathrm{v}(s)=(x(s), y(\mathrm{~s}))$ and its energy functional can be written as: 


$$
E=\int_{0}^{l} E_{\text {int }}(v(s)) d s+0 \int_{\text {imagd }}^{l} E_{\text {ing }}(v(s)) d s+\int_{0}^{l} E_{\text {ext }}(v(s)) d s
$$

Where $E_{\text {int }}$ represents the spline internal energy and $E_{\text {image }}$ represent the image forces and $E_{\text {ext }}$ represent to the external forces. The spline energy controlled by $a(s)$ and by $B(\mathrm{~s})$. Therefore, the internal spline energy can be written as:

$$
E_{\mathrm{int}}=\frac{\left(a(s)\left|v_{S}(s)\right|^{2}+B(s)\left|v_{s S}(s)\right|^{2}\right)}{2}
$$

The total image energy can be represented as a combination of three weighted energy functions and can be written as:

$$
E_{\text {image }}=w_{\text {line }} E_{\text {line }}+w_{\text {edge }} E_{\text {edge }}+w_{\text {term }} E_{\text {term }}
$$

Active contour models (Snakes) can be represented by two models: region based models and edge-based models. The characteristics of the image determine the model we should choose. The main advantage of snakes models is the ability of snakes to give a linear description of the object shape during the time of convergence without adding extra processing. But what scientifically limits the use of snakes is the need of the method to have strong image gradients to be able to drive the contour.

\subsection{Threshold Method}

Thresholds in these algorithms can be selected manually according to a priori knowledge or automatically through image information. Algorithms can be further divided to edge-based ones, region-based ones and hybrid ones (Canny, 1986; Gonzalez et al., 2002; Mukherjee et al., 2004; Sezgin et al., 2004). Thresholds in the edge-based algorithms are related with the edge information. Structures are depicted by edge points. Common edge detection algorithms such as Canny edge detector and Laplacian edge detector can be classified to this type. Algorithms try to find edge pixels while eliminate the noise influence. For example, Canny edge detector uses the threshold of gradient magnitude to find the potential edge pixels and suppresses them through the procedures of the non maximal suppression and hysterics shareholding. As the operations of algorithms are based on pixels, the detected edges are consisted of discrete pixels and therefore may be incomplete or discontinuous. Hence, it is necessary to apply postprocessing like morphological operation to connect the breaks or eliminate the holes. The method has the ability to segment 3D image with good accuracy, but the disadvantage of this method is the difficulty of the method to process the images of textured blob objects.

\subsection{Topological Alignments Method}

Topological Alignments Method depends on formalize the problem of aligning two consecutive frames as a generalized assignment problem (Miura et al., 2005; Danuser et al., 2005; Zimmer et al., 2006; Ersoy et al., 2007). The algorithm links the segmentation of two frames from the video sequence. The process starts from the output of the segmentation procedure, the algorithm work by finding the maximum weighted solutions to a generalized matching between two segments, and derive weights from relative sets of segments.

In this method we identify the segmentation of the first image into $m$ segments with an index set $P=\{1, \ldots, m\}$, and the second image segmentation into $n$ segments with an index set $Q=\{1, \ldots$, $n$ \}. Then, alignments between these sets can be introduced through partitioning $P$ and $Q$ into an equal number of subsets. 
Assuming that cells move moderately between two consecutive frames, we assign the relative overlap of $p$ and $q$ as their weight, formally defined as

$w(p, q):=|A(p) \cup A(q)| /|A(p) \cup A(q)|$

In general, we consider the sets of segments which have overlap close to 1 as one cell, but the sets of segments which have overlap close to 0 not to be considered as one cell. Based on these weights, we can consider the notions of the topological alignments. We denote $\boldsymbol{P L}(M)$ for the set of all L-partitioning`s of a finite set $M$; note that given a partition $S \in P L(M)$, we consider $S$ as a family of sets and hence can identify the L subsets by writing $S=(S 1, \ldots, S \mathrm{~L})$. This allows us to state our alignment as finding those partitioning`s $S$ and $T$ that realize the maximum in the target function.

The topological alignments method improves the performance of segmentation of cell tracking by explicitly taking into account the inherent problems of over (one cell is split into two segments) and under (one segment fully covers two cells) segmentation, while still allowing the detection of cell division. The algorithm links segments between every frame and the next one, that will reduce the number of false trajectories and false detections. The method can deal with low contrast images and shape cells and improves the filtration efficiency. The advantage of the method is the ability of topological alignments to mitigate the image noise and cell deformation.

\subsection{Watersheds Method}

Watershed image segmentation is based on the theory of Mathematical Morphology (Beucher et al., 1979; Najman and Schmitt, 1996; Meyer et al., 1996; Lezoray et al., 2003; Huguet et al., 2004). Numerous techniques have been proposed to compute watersheds. The classical idea for building the watershed is using a geographical analogy, begin by piercing the regional minima of the surface. Then slowly immerse the image into a lake. The water progressively floods the basins corresponding to the various minima. To prevent the merging of two different waters originating from two different minima, we erect a dam between both lines. Once the surface is totally immersed, the set of the dams thus built is the watershed of the image. In one dimension, the location of the watershed is straightforward: it corresponds to the regional maxima of the function. In two dimensions, one can say in an informal way that the watershed is the set of crest lines of the image, emanating from the saddle points.

The method stick this initial contour to the maximum contained watershed contour. For label image $G=[R, E]$, we assume each edge eij $C E$ is a directing curve with the direction the same as clockwise direction of region $r i$ s contour.

Watershed contour discriminate to initial contour. A watershed contour is also a closed contour, but it is exactly along the watershed edges, i.e., it is composed of watersheds. When we input an initial manually delineated contour, we need to push (or expend) it to the nearby watershed edge to facilitate the later calculation. The method helps to improve the capture range but it has disadvantage of over segmentation. 
International Journal of Computer Science \& Information Technology (IJCSIT) Vol 3, No 5, Oct 2011

Table 2.1 Comparison of the Image Segmentation Methods.

\begin{tabular}{|c|c|c|}
\hline Method & Advantage & Disadvantage \\
\hline Inverse dynamics method & $\begin{array}{l}\text { - Data are very good. } \\
\text { - Animation is of high } \\
\text { quality. } \\
\text { - Using a nonlinear } \\
\text { optimizer. }\end{array}$ & $\begin{array}{l}\text { - Many different EMG } \\
\text { patterns can produce the } \\
\text { same kinematic output }\end{array}$ \\
\hline Active contour method & $\begin{array}{l}\text { - Use active contour } \\
\text { models } \\
\text { - Preserves global line } \\
\text { shapes efficiently }\end{array}$ & $\begin{array}{l}\text { - Should find strong image } \\
\text { gradients to drive the } \\
\text { contour. } \\
\text { - Lacking accuracy with } \\
\text { weak image boundaries } \\
\text { and image noise }\end{array}$ \\
\hline Watersheds Method & $\begin{array}{l}\text { - } \quad \text { Based on Mathematical } \\
\text { Morphology } \\
\text { - Helps to improve the } \\
\text { capture range }\end{array}$ & - Over segmentation \\
\hline $\begin{array}{l}\text { Novel edge-based } \\
\text { method }\end{array}$ & $\begin{array}{l}\text { - Algorithm based on an } \\
\text { energy minimization } \\
\text { procedure. }\end{array}$ & $\begin{array}{l}\text { - depends on the } \\
\text { assumption that the } \\
\text { deformation and } \\
\text { movement of the tracked } \\
\text { object is small between } \\
\text { the frames. }\end{array}$ \\
\hline $\begin{array}{l}\text { Topological alignments } \\
\text { method }\end{array}$ & $\begin{array}{l}\text { - Improve the filtration } \\
\text { efficiency. } \\
\text { Using linkage } \\
\text { clustering. }\end{array}$ & - Complicated. \\
\hline $\begin{array}{l}\text { Pattern Recognition } \\
\text { method }\end{array}$ & $\begin{array}{l}\text { Pattern recognition } \\
\text { fields used to perform } \\
\text { the segmentation. } \\
\text { - The method used to } \\
\text { model relationships } \\
\text { between inputs and } \\
\text { outputs. }\end{array}$ & $\begin{array}{l}\text { - Restrictions on shape } \\
\text { parameters. } \\
\text { - Complicated }\end{array}$ \\
\hline Threshold method & $\begin{array}{l}\text { Try to find edge pixels } \\
\text { while eliminate the } \\
\text { noise influence. } \\
\text { Use gradient } \\
\text { magnitude to find the } \\
\text { potential edge pixels. }\end{array}$ & $\begin{array}{l}\text { - The detected edges are } \\
\text { consisted of discrete } \\
\text { pixels and may be } \\
\text { incomplete or } \\
\text { discontinuous. } \\
\text { Computationally } \\
\text { expensive }\end{array}$ \\
\hline
\end{tabular}




\section{Conclusions}

From the previous review, we classify the current methods and summarize their features. Also each method has its suitable application fields, and researchers should combine the application background and practical requirements to design proper algorithms. Accuracy, complexity, efficiency and interactivity of a segmentation method should all be the considered factors.

This paper makes a review on the current segmentation methods, and the main tendency of each method with their principle ideas, application field, advantages and disadvantages are discussed.

\section{REFERENCES}

[1] S. Osher and J. Sethian, "Fronts propagating with curvature dependent speed: Algorithms based on Hamilton - jacobi formulations," Journal of Computationl Physics, pp. 12-49, 1988.

[2] Bouguet, J. (2000). Pyramidal implementation of the Lucas Kanade feature tracker. Intel Corporation Microprocessor Research Labs: OpenCV Documents.

[3] Bradski, G. (2000). The Open CV Library. Dr. Dobb's Software Tools for the Professional Programmer.

[4] Coskun, H., Li, Y., and Mackey, M. A. (2007). Ameboid cell motility: A model and inverse problem, with an application to live cell imaging data. Journal of Theoretical Biology, 244(2): $169-179$.

[5] Kass, M., Witkin, A., and Terzopoulos, D. (1987). Snakes: Active contour models. International Journal of Computer Vision, pages 321-331.

[6] Li, K., Miller, E., Weiss, L., Campbell, P., and Kanade, T. (2006). Online tracking of migrating and proliferating cells imaged with phase - contrast microscopy. Proc. Of the 2006 Conf, on Computer Vision and Pattern Recognition Workshop (CVPRW'06), pages 65-72.

[7] Mukherjee, D., Ray, N., and Acton, S. (2004). Level set analysis for leukocyte detection and tracking. IEEE Trans Image Process, 13(4):562-72.

[8] Ray, N., Acton, S., and Ley, K. (2002). Tracking leukocytes in vivo with shape and size constrained active contours. 21(10):1222-1235.

[9] Smart, J., Hock, K., and Csomor, S. (2005). Cross-Platform GUI Programming with wxWidgets. Prentice Hall PTR.

[10] Zimmer, C., Labruyre, E., Meas-Yedid, V., Guilln, N., and Olivo-Marin, J. (2002). Segmentation and tracking of migrating cells in videomicroscopy with parametric active contours: a tool for cell-based drug testing. IEEE Trans Med Imaging, 21(10):1212-21.

[12] Shtern F. Digital mammography and related technologies: a perspective from the National Cancer Institute. Radiology 1992; 183:629-630.

[13] Feig SA, Yaffe MJ. Current status of digital mammography. Semin Ultrasound CT MR 1996; 17:424-443.

[14] Aylward SR, Hemminger BM, Pisano ED. Mixture modeling for digital mammogram display And analysis. In: Karssemeijer N, Thijssen M, Hendriks J, van Erning A, eds. Digital mammography Nijmegen, 1998. Dordrecht, the Netherlands: Kluwer Academic, 1998; 305-312.

[15] Pisano ED, Zong S, Hemminger BM, et al. Contrast limited adaptive histogram equalization image processing to improve the detection of simulated spiculations in dense mammograms. $\mathrm{J}$ Digit Imaging 1998; 11:193-200.

[16] Chan HP, Vyborny CJ, MacMahon H, et al. Digital mammography ROC studies of the effects of pixel size and unsharp - mask filtering on the detection of subtle microcalcifications. Invest Radiol 1987; 22:581-589. 
International Journal of Computer Science \& Information Technology (IJCSIT) Vol 3, No 5, Oct 2011

[17] Byng JW, Critten JP, Yaffe MJ. Thickness equalization processing for mammographic images. Radiology 1997; 203:564-568.

[18] Bick U, Giger ML, Schmidt RA, Nishikawa RM, Doi K. Density correction of peripheral breast tissue on digital mammograms. RadioGraphics 1996; 16:403-411.

[19] Nath SK, Bunyak F, Palaniappan K: Robust Tracking of Migrating ells Using Four- Color Level Set Segmentation. ACIVS 2006:920-932.

[20] Koehler A, Schambony A, Wedlich D: Wnt Signaling in Embryonic Development Elsevier 2007 chap. Cell migration under control of Wnt signaling in the vertebrate embryo:159-201.

[21] Zimmer C, Zhang B, Dufour A, Thebaud A, Berlemont S, Meas-Yedid V, O Marin JC: On the Digital Trail of Mobile Cells. Signal Processing Magazine 2006, 23(3):54-62.

[22] Palaniappan K, Ersoy I, Nath SK: Moving Object Segmentation Using the Flux Tensor for Biological Video Microscopy. Lect Notes Comput Sci. 2007, 4810(LNCS):483-493.

[23] Miura K: Tracking Movement in Cell Biology. Advances in Biochemical Engineering/ Biotechnology 2005, 95:267-295.

[24] Meijering E, Smal I, Danuser G: Tracking in molecular bioimaging. Signal Processing Magazine, IEEE 2006, 23(3):46-53.

[25] Bouguet, J. (2000). Pyramidal implementation of the Lucas Kanade feature tracker. Intel Corporation Microprocessor Research Labs: OpenCV Documents.

[26] Bradski, G. (2000). The Open CV Library. Dr. Dobb's Software Tools for the Professional Programmer.

[27] Kass, M., Witkin, A., and Terzopoulos, D. (1987). Snakes: Active contour models. International Journal of Computer Vision, pages 321-331.

[28] Li, K., Miller, E., Weiss, L., Campbell, P., and Kanade, T. (2006). Online tracking of migrating and proliferating cells imaged with phase - contrast microscopy. Proc. of the 2006 Conf, on Computer Vision and Pattern Recognition Workshop (CVPRW’06), pages 65-72.

[29] Mukherjee, D., Ray, N., and Acton, S. (2004). Level set a nalysis for leukocyte detection and tracking. IEEE Trans Image Process, 13(4):562-72.

[30] Dzyubachyk O, Niessen W, Meijering E: Advanced Level - Set Based Multiple - Cell Segmentation and Tracking in Time - Lapse Fluorescence Microscopy Images. In IEEE International Symposium on Biomedical Imaging: From Nano to Macro Edited by: OlivoMarin JC, Bloch I, Laine A. IEEE, Piscataway, NJ; 2008:185-188.

[31] Li Y, Zheng Y, Doermann D, Jaeger S: Script-Independent Text Line Segmentation in Freestyle Handwritten Documents. IEEE Trans Pattern Anal Mach Intell. 2008, 30(8):1313-1329.

[32] Ersoy I, Bunyak F, Mackey M, Palaniappan K: Cell Segmentation Using Hessian - Based Detection and Contour Evolution with Directional Derivatives. International Conference on Image Processing 2008:1804-1807.

[33] Tseng Y, Kole T, Wirtz D: Micromechanical Mapping of Live Cells by Multiple Particle Tracking Microrheology. Biophysical Journal 2002, 83(6):3162-3176.

[34] Apgar J, Tseng Y, Fedorov E, Herwig M, Almo S, Wirtz D: Multipleparticle tracking measurements of heterogeneities in solutions of actin filaments and actin bundles. Biophysical Journal 2000, 79(2):1095-1106.

[35] Schütz GJ, Schindler H, Schmid T: Single-molecule microscopy on model membranes reveals anomalous diffusion. Biophysical Journal 1997, 73:1073-1080.

[36] Anderson C, Georgiou G, Morrison I, Stevenson G, Cherry R: Tracking of cell surface receptors by fluorescence digital imaging microscopy using a charge-coupled device camera. Low-density lipoprotein and influenza virus receptor mobility at 4 degrees C. Journal of Cell Science 1992, 101(2):415-425. 